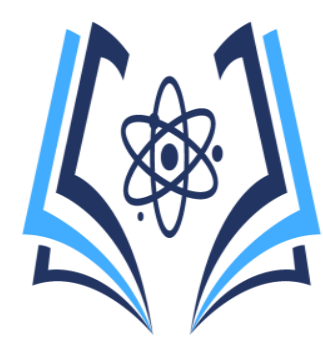

\title{
Proceso de atención de enfermería en pacientes con hipertensión arterial
}

\section{Nursing care process in patients with hypertension}

\section{Proceso de atención de enfermería}

\author{
Maribel Celi Vásquez Paucar ${ }^{1}$ \\ Dolores Mirella Cedeño Holguín ${ }^{2}$ \\ ${ }^{1}$ Universidad Estatal del Sur de Manabí, maribel.vasquez@unesum.edu.ec, código ORCID: \\ https://orcid.org/0000-0002-8835-5273
}

Contacto: maribel.vasquez@unesum.edu.ec

Recibido: 29-09-2019

Aprobado: 15-12-2019

\begin{abstract}
Resumen
El presente trabajo hace referencia al proceso de atención de enfermería, el que es considerado como la herramienta indispensable para garantizar una adecuada intervención médica en personas que padecen hipertensión (o tensión arterial alta), este padecimiento es un trastorno grave que incrementa de manera significativa el riesgo de sufrir cardiopatías, encefalopatías, nefropatías y otras enfermedades, por lo cual se ha considerado como uno de los problemas más graves que se da a nivel mundial, llegando a afectar a más de mil millones de personas, donde la mayoría de ellos muere. El objetivo fue estudiar el papel de los cuidados en enfermería en pacientes con hipertensión arterial para el conocimiento de los factores de riesgo asociados a esta enfermedad, la metodología aplicada estuvo fundamentada en bibliografías relevantes, la que brindo información necesaria para fundamentar la investigación, ya que proporciona pautas con la finalidad de controlar y mejorar la hipertensión arterial en los adultos. El resultado obtenido después de la investigación se fundamenta en la elaboración de un plan de cuidados, aplicado a los adultos mayores que padecen hipertensión arterial. Por lo descrito se concluye que, mediante la investigación desarrollada fue posible conocer científicamente lo que engloba la hipertensión arterial y los principales factores de riesgo que causan esta enfermedad en la población adulta, así como cuáles son los efectos que la causan.
\end{abstract}

Palabras clave: Plan, cuidados, sistema, enfermos, presión.

\section{Abtract.}

This work refers to the nursing care process, which is considered an essential tool to guarantee adequate medical intervention in people suffering from hypertension (or high blood pressure), this condition is a serious disorder that significantly increases the risk of suffering from heart disease, encephalopathy, nephropathy and other diseases, for which it has been considered one of the most serious problems that occurs worldwide, affecting more than one billion people, where most of them die. The objective was to study the role of nursing care in patients with arterial hypertension for the knowledge of the risk factors associated with this disease, the applied methodology was based on relevant bibliographies, which provides the necessary information to support the research, as which provides guidelines for the purpose of controlling and improving high blood pressure in adults. The result obtained after the research is based on the development of a care plan, applied to older adults with high blood pressure. Based on what has been described, it is concluded that, through the research carried out, it was possible to scientifically know what is encompassed by arterial hypertension and the main risk factors that cause this disease in the adult population, as well as what are the effects that cause it.

Keywords: Plan, care, system, patients, pressure.

\section{Introducción}

La principal causa de muerte en Latinoamérica es producto de enfermedades cardiovasculares, entre 1968 y 1987, el índice de muertes anuales debido a esta enfermedad incrementó del 20 al 27\%, especialmente en hombres. Las enfermedades cardiovasculares son las responsables de un tercio de muertes a nivel mundial, sin embargo, para reducir este tipo de patología se requiere estrategias como: modificaciones en el estilo de vida, las cuales deben incluir una dieta saludable, actividad física, 
disminuir el hábito de fumar, para así prevenir la hipertensión, diabetes e hiperlipidemia. (Perez, 2017)

Según la OMS (Organizacion Mundial de la Salud, 2019 ) en el 2015, 1 de cada 4 hombres y 1 de cada 5 mujeres tenían hipertensión, donde solo el 1 de cada 5 personas hipertensas tenían controlado el problema, por los cuales una de las principales metas propuestas a nivel mundial para dicha patología es llegar a su reducción en un 25\% para el 2025 con respecto a los valores que se obtuvieron de referencia en 2010.

Se estima que en el mundo hay 1130 millones de personas con hipertensión, y casi la totalidad de ellas (cerca de dos tercios) vive en países de ingresos bajos y medianos, sobre todo relacionados a los países americanos, sobre todo las Américas del sur, mientras que en los países desarrollados no se espera que ocurra un incremento tan drástico en relación a enfermedades cardiovasculares.

La mayoría de los países Latinoamericanos tiene una mortalidad cardiovascular que representa entre el 11.6 y el $43.5 \%$ de la mortalidad general, siendo la primera causa de muerte en la mayoría de ellos, debido a que muchos de ellos no conocen su condición, por lo cual los procesos de atención de enfermería se ha vuelto el punto clave para que ellos puedan llegar a mejorar su calidad de vida.

Según el Estudio Epidemiológico Prospectivo Urbano Rural (CONSEJO NACIONAL DE CIENCIA Y TECNOLOGÍA (CONACYT), 2019) sobre hipertensión (PURE, por sus siglas en inglés) en Argentina, Brasil y Chile, de forma combinada, sólo el 57\% de los hipertensos conoce su condición, únicamente el 53\% de ellos está bajo tratamiento, y sólo el $30 \%$ de los tratados tiene su presión arterial controlada, en el año 2019 se buscó que al menos el $35 \%$ de las personas con hipertensión tuvieran la presión arterial bajo control. Hasta ahora, sólo tres países superan esa meta: Canadá (68\%), los Estados Unidos $(52 \%)$, y Cuba $(36 \%)$, el resto tendrá que hacer un poco más de esfuerzos para conseguirla.

En América Latina, Ecuador es el país con la mayor prevalencia de hipertensión arterial y cuya primera causa de muerte son las enfermedades cardiovasculares secundarias. Incluso un $46 \%$ de la población ecuatoriana posee hipertensión arterial y, lo devastador del asunto es que solamente un $15 \%$ de esa población que es hipertensa tiene un control adecuado de sus cifras de presión arterial.

"En el Ecuador el 9,3\% de la población afectada son a partir 18 a 59 años de edad, además es considerada como uno de los principales factores de riesgo desencadenante de un significativo número de padecimientos cerebrovasculares, cardiovasculares y renales" (Cabrera Perez, 2017).

"La hipertensión arterial y sus complicaciones como insuficiencia renal, ceguera, infartos, derrames cerebrales, entre otros, constituyeron en el 2010 la primera causa de mortalidad en el Ecuador. En territorios como Manabí, Esmeraldas, Cañar, Los Ríos, Azuay y Napo existe mayor incidencia de esta enfermedad" (Ministerio de Salud Publica , 2017).

La falta de conocimiento en la mayoría de la población afectada provoca la causa de muerte, acentuando así la importancia del actuar del profesional de enfermería para el debido control y mejora de la calidad de vida de los pacientes hipertensos, estableciéndose diversas estrategias en los diferentes niveles de atención en el personal de salud para poder esquivar graves complicaciones en la población afectada, ya que son los involucrados directos para una detección oportuna, capacitación al paciente así como sus cuidados específicos y tratamiento garantizando al paciente una mejor calidad de vida (Pérez, 2017).

Florence Nightingale nació en Florencia, Italia el 12 de mayo de 1820 considerada como la pionera de la enfermería su teoría gira en torno a la relación del paciente con su entorno, la relación de la enfermera con él paciente y la relación de la enfermera con el entorno del paciente. (Young, Hortis de Smith, Chambi , \& Finn, 2011)

La teoría de Marjory Gordon aplicable en el PEA deriva de los patrones funcionales establecidos por la misma. Gordon estableció su teoría en base de los 11 patrones o dominios funcionales; los mismos que ayudan a valorar alteraciones de salud que afectan al individuo y a su vez brinda una valoración de enfermería organizada y planificada que busca obtener datos subjetivos y objetivos del paciente los mismos que ayudan a establecer los diagnósticos enfermeros ya sean reales o potenciales, sus patrones se establecieron en la NANDA. (Cisne, 2020)

Para Virginia Henderson, el individuo sano o enfermo es un todo completo, que presenta catorce necesidades fundamentales y el rol de la enfermera consiste en ayudarle a recuperar su independencia lo más rápidamente posible.

Necesidad de respirar, de beber y comer, de eliminar, de moverse y mantener una buena postura, de dormir y descansar, de vestirse y desvestirse, de mantener la temperatura corporal dentro de los límites normales, de estar limpio, aseado y proteger sus tegumentos, de evitar los peligros, de comunicarse según sus creencias y sus valores, de ocuparse para realizarse, de recrearse y de aprender. (línea, 2016)

Proceso de atención de enfermería 
El proceso de atención (PEA) es un método para promover un cuidado reflexivo, individualizado y orientado buscando resultados. Su uso en un entorno educativo está relacionado con la promoción del desarrollo profesional, acompañado de la teoría de la asignatura y pautas de enseñanza. (Saniabra \& Pérez, 2013)

Guía para el trabajo profesional, científico, sistemático y humanístico en la práctica de la enfermería, centrada en la evaluación simultánea de los avances y cambios en el estado de salud de los individuos, familias y / o grupos a partir de la enfermería, concedido por enfermeras. Se caracteriza por tener validez universal y relación directa paciente- profesional.

Uno de sus objetivos es formar una herramienta de trabajo para el personal de enfermería y realizar de forma continua investigaciones sobre la misma

\section{Diagnóstico de enfermería}

Esta etapa incluye el proceso de análisis y síntesis para emitir juicios y conclusiones sobre la salud de la persona frente a necesidades o problemas de salud reales o potenciales. Una vez determinado, se debe considerar la estructura del PES (Problemas, Etiología y Signos / Síntomas).

\section{Intervenciones de enfermería}

Las intervenciones (actividades o acciones de enfermería) están diseñadas para alcanzar las metas del plan, en el proceso de enfermería se deben definir las intervenciones necesarias para lograr los estándares de resultado previamente establecidos, por lo que la intervención común implica varias acciones. Las Intervenciones de Enfermería pueden ser directas o indirectas.

\section{Plan de cuidados de enfermería}

Es un convenio de medidas asistenciales basado en las necesidades de cuidados de un grupo de pacientes con un mismo diagnóstico médico, una vez implantado y monitorizado. Estos planes se convierten en herramientas de referencia en la memoria colectiva del equipo de enfermería y son elementos esenciales para garantizar la relevancia, la continuidad en la atención de enfermería y la individualización. (Abad, 2016)

\section{Factores de la intervención de enfermería}

Al elegir una intervención, se deben considerar seis factores:

Resultados deseados en el paciente, características del diagnóstico de enfermería, investigación sobre la intervención, factibilidad para realizar la intervención, aceptación por el paciente y capacidad del profesional de enfermería.

\section{Niveles de atención}

Atención primaria es lo más cercano a la población, es decir, el grado de primer contacto o la puerta de entrada al sistema de salud, en la atención secundaria se ubican servicios relacionados con la medicina interna, pediatría, obstetricia y ginecología, cirugía general y psiquiatría y la atención terciaria se reserva para tratar algunos problemas habituales, donde se resuelven alrededor del $5 \%$ de los problemas de salud. (Vignolo, Vacarezza, Álvarez, \& Sosa, 2015)

\section{Tratamiento}

El tratamiento enfermero para la administración de medicamentos del paciente debe ser bajo las reglas de seguridad establecidas, integrando la aplicación de los 15 correctos:

Higienización de los medicamentos antes de su preparación y administración, conocer los antecedentes alérgicos del paciente, tomar los signos vitales antes de la administración de medicamentos, verificar que sea el medicamento correcto, la dosis correcta, la vía de administración correcta, la hora correcta, el paciente correcto, la técnica de administración correcta, la velocidad de infusión correcta, también se verifica la fecha de caducidad del medicamento antes de su administración, no se deben administrar medicamentos bajo órdenes verbales, se debe registrar la administración de medicamentos minutos antes de ser aplicados, el enfermero deberá preparar y administrar el mismo los medicamentos y por último se educa tanto al paciente como a su familia sobre la correcta administración de los fármacos.

\section{Presión arterial}

La sangre impulsada por el corazón fluye a través del torrente sanguíneo o más preferiblemente a través del sistema arterial y se somete a una presión llamada tensión arterial, se mide en $(\mathrm{mmHg})$, teniendo la presión arterial sistólica (PAS), que está determinada por el impulso cardíaco, y se conoce como alta y está la presión arterial diastólica (PAD) baja, que depende de la resistencia de las arterias al paso de la sangre.

\section{Hipertensión arterial}

La hipertensión es una patología crónica en la que existe una alta presión persistente en los vasos sanguíneos, que puede dañar a los mismos. Cuanto mayor sea la tensión, más esfuerzo debe poner el corazón.

La hipertensión se define como presión arterial sistólica cuando en un paciente es superior a 140 mmHg y la presión arterial diastólica cuando es superior a $90 \mathrm{mmHg}$, y hoy en día es un problema de salud pública en todo el mundo. (Montes de Oca, Guerra, Castañeda, \& Coma, 2019) 


\section{Etapas de la hipertensión arterial}

La hipertensión elevada se da cuando hay un aumento de la presión arterial es una presión sistólica entre 120 y $129 \mathrm{mmHg}$ y una presión diastólica por debajo de $80 \mathrm{mmHg}$.

Se considera a la hipertensión en etapa 1 cuando esta está entre 130 y 139 mmHg la sistólica y la presión arterial diastólica está entre 80 y $89 \mathrm{~mm} \mathrm{Hg}$.

La hipertensión en etapa 2 es una forma más grave de hipertensión con una presión sistólica de 140 $\mathrm{mmHg}$ o superior, y una presión diastólica de 90 $\mathrm{mmHg}$ o superior.

La hipertensión en estadio o etapa 3 es más grave, considerándose una presión arterial sistólica igual o superior a $180 \mathrm{mmHg}$ y una presión arterial diastólica igual o superior a $110 \mathrm{mmHg}$. (yo, 2017)

\section{Clasificación de la hipertensión arterial}

La hipertensión arterial (HTA) primaria básica o mayor oscila entre el $85-90 \%$ de los casos. En su origen se incluye la obesidad, el estilo de vida sedentario, el consumo excesivo de alcohol, el tabaquismo y muchos otros factores mientras que la HTA secundaria se representa entre el $0-15 \%$ de los casos. Generalmente causado por malformaciones de arterias, riñones o aorta; y problemas hormonales (corteza suprarrenal o bulbo raquídeo, glándula pituitaria, tiroides, etc.).

\section{Causas de la hipertensión arterial}

La característica básica de la hipertensión (HTA) es la presencia de disfunción endotelial (DE) y del equilibrio entre los factores de los vasos sanguíneos (óxido nítrico NO, factor hiperpolarizante endotelioEDHF). (Wagner-Grau, 2016)

Uno de cada 20 casos de hipertensión es causado por una enfermedad o medicación subyacente. La insuficiencia renal crónica (ERC) es la causa más común de presión arterial alta porque los riñones no filtran el líquido que se conoce como orina. Este exceso de líquido puede provocar la hipertensión arterial.

El género es una variable que atraviesa todos los determinantes sociales de la salud, es un atributo que configura la salud de hombres y mujeres, según su posición en la jerarquía de poder, prestigio y adquisición de recursos. (Deborah, Pereyra, Cófreses, \& Dorina, 2015)

Los estudios epidemiológicos también han demostrado que tanto los negros como los asiáticos tienen tendencia a sufrir el llamado síndrome de resistencia a la insulina, en el que la insulina deja de funcionar y conduce a la obesidad abdominal y a la dislipidemia, que es un factor de la HTA. Esto también puede explicar la mayor incidencia de enfermedades cardiovasculares entre estas personas. (https://secardiologia.es/comunicacion/notas-deprensa/notas-de-prensa-sec/4293-raza-condicionariesgo-, 2014)

\section{Consecuencias de la hipertensión arterial}

El retraso del crecimiento intrauterino (RCIU) está relacionado con los factores de riesgo de la madre y las causas de la placenta y la dependencia fetal. La aparición de esta patología es relevante porque se relaciona con la morbilidad y mortalidad de los recién nacidos y la incidencia de patología crónica en la vida adulta a corto plazo. (Rybertt, Azua, \& Rybertt, 2016)

\section{Tratamiento de la hipertensión arterial}

El tratamiento de la hipertensión se puede realizar de dos formas, una es para desarrollar un estilo de vida saludable y la otra es a través de medicamentos.

Tratamiento no farmacológico de la hipertensión incluye la dieta, el ejercicio y el abandono del tabaco

En el tratamiento farmacológico hay 6 tipos de medicamentos que se utilizan para tratar la HT: que son los diuréticos, bloqueantes beta, antagonista de los canales de calcio, inhibidores de la enzima convertidora de angiotensina (IECA), antagonistas del receptor de angiotensina II (ARA-II) y bloqueadores alfa adrenérgicos o alfa Bloqueador.

Los parámetros hemodinámicos en los pacientes críticos son de ayuda para el diagnóstico, mientras que los cambios fisiológicos en algunos casos dependen del aumento de la edad. (Aguilar \& Vega, 2020)

El apoyo familiar es un factor clave en el tratamiento de los pacientes hipertensos. En términos de seguimiento y control de la salud se debe fomentar una actitud personal positiva favoreciendo a la comunicación familiar que brindará ayuda o asistencia en tiempos de crisis y promover la atención al paciente. Condiciones de salud, como hábitos alimenticios, ejercicio físico y persistencia o persistencia en la toma de medicamentos antihipertensivos. Es importante señalar que sólo los pacientes con sus familias pueden promover la paz y la fortaleza, hacerlos sentir seguros, protegidos, y alentarlos a buscar soluciones para mejorar su salud y disminuir sus cifras de presión arterial.

\section{Materiales y métodos}

El método en el que se basó esta investigación es el cualitativo, porque esta metodología implementa técnicas como la observación y grupos de discusión.

En este trabajo se aplicó, la investigación informativa o expositiva y la técnica fue bibliográfica, porque permitió al investigador seleccionar y analizar producción científica, como 
son: libros, artículos acreditados, revistas indexadas y demás documentos autorizados e influyentes, relacionados al proceso de atención de enfermería e hipertensión arterial.

Población y muestra: El total de la población hipertensa a nivel mundial es de 1130 millones de personas, de la cual se tomó como muestra el 9,3\% de los pacientes adultos del Ecuador que padecen esta patología.

\section{Criterios de inclusión y exclusión}

Se consideraron los siguientes juicios:

Los criterios de inclusión son aquellas características que necesariamente deberán tener los elementos de estudio (muestra) como:

> La cantidad de pacientes adultos que fallecen a diario a causa de la hipertensión arterial.

$>$ Las consecuencias que pueden desencadenar en los adultos la hipertensión arterial, afectando a otros órganos como el riñón y los ojos.

$>$ Debido a que la mayor cantidad de pacientes con hipertensión arterial son adultos, teniendo mas prioridad durante la investigación.

$>\mathrm{Al}$ ser pacientes adultos corren un mayor riesgo de padecer hipertensión arterial, la cual se puede complicar con el paso de los años reduciendo la probabilidad de vida en los pacientes.

Por su parte los criterios de exclusión son aquellos elementos de estudio que no presentan las características de la muestra como:

$>$ El número de pacientes jóvenes que padecen hipertensión arterial.

$>$ Al conocer que una de las causas de la hipertensión arterial es la edad, se excluye a los jóvenes durante la investigación.

$>$ Identificando que la mayor cantidad de los jóvenes de entre 18 y 22 años lleva una vida activa en la que incluye ejercicio y una dieta, se estima que tienen una menor incidencia en padecer cierta patología, debido a que las complicaciones se ven en un futuro.

\section{Resultados}

Los resultados obtenidos de la investigación realizada, tributan al incremento de conocimientos en elaboración, desarrollo y aplicación del proceso de atención de enfermería, empezando con la primera etapa que es la valoración, la que proporciona todos los datos e información del paciente, después se realizan las intervenciones y finalmente se obtienen los resultados, asi como de los planes de cuidado aplicados a pacientes con HTA, los que presentan riesgos, necesitan cuidados y requieren educación en salud. En base a lo aprendido y para mejorar los procesos de atención, se presenta una idea de plan de cuidados para los pacientes que padecen Hipertensión arterial.

\section{Propuesta del plan de cuidados en paciente hipertenso}

Establecimiento: hospital General Jipijapa

Nombre: Elvia Elizabeth Cedeño Guaranda

Edad: 54 años

Historia clínica: 01707

Fecha: 20/08/2020

Diagnóstico: Paciente sexo femenino hipertenso

Días de intervenciones: 5 días

\begin{tabular}{|c|c|c|c|}
\hline Diagnóstico & Resultados esperados & Intervenciones de enfermería (NIC) & Evaluación \\
\hline $\begin{array}{l}\text { Clase: } 0001 \text { Toma de } \\
\text { conciencia de la salud } \\
\text { Reconocimiento del } \\
\text { bienestar y funcionamiento }\end{array}$ & $\begin{array}{l}\text { Obtener en el paciente un } \\
\text { manejo adecuado y } \\
\text { responsable con respecto al } \\
\text { cuidado y bienestar de su } \\
\text { salud. } \\
\text { En } 5 \text { días }\end{array}$ & $\begin{array}{l}\text { Ampliar los conocimientos del } \\
\text { paciente de cuán importante es } \\
\text { mantener una vida saludable y por } \\
\text { ende un funcionamiento normal. } \\
\text { 1. Preparar el material. } \\
\text { 2. Proporcionar información } \\
\text { al paciente de los tipos de }\end{array}$ & $\begin{array}{lr}\text { El paciente cambió } & \text { su } \\
\text { perspectiva de cuidado en la } & \\
\text { salud, obedeció los } & \text { del } \\
\text { requerimientos } & \text { profesional enfermero, } \\
\text { empezó una vida diferente } & \text { llena de bienestar, con más }\end{array}$ \\
\hline
\end{tabular}




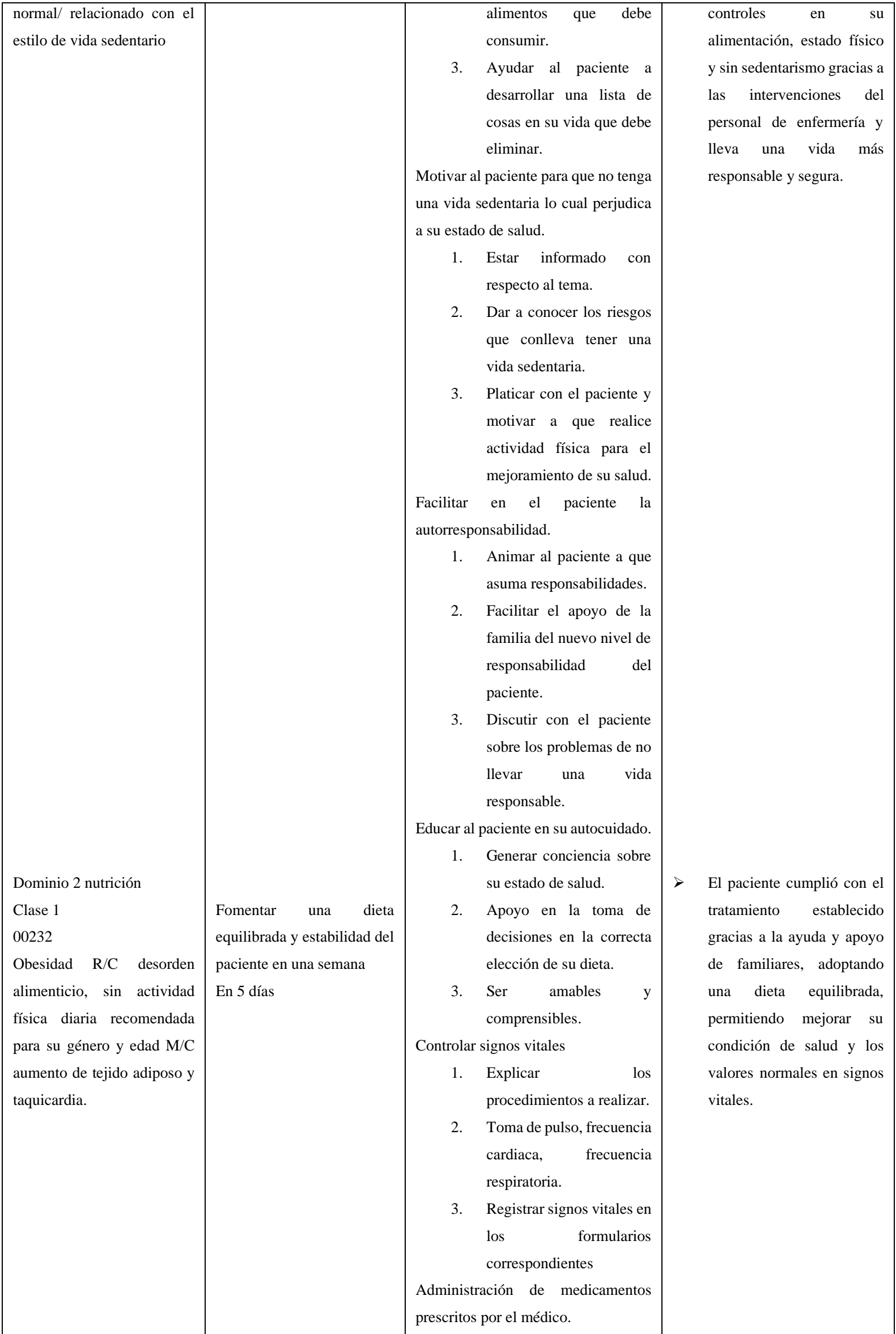




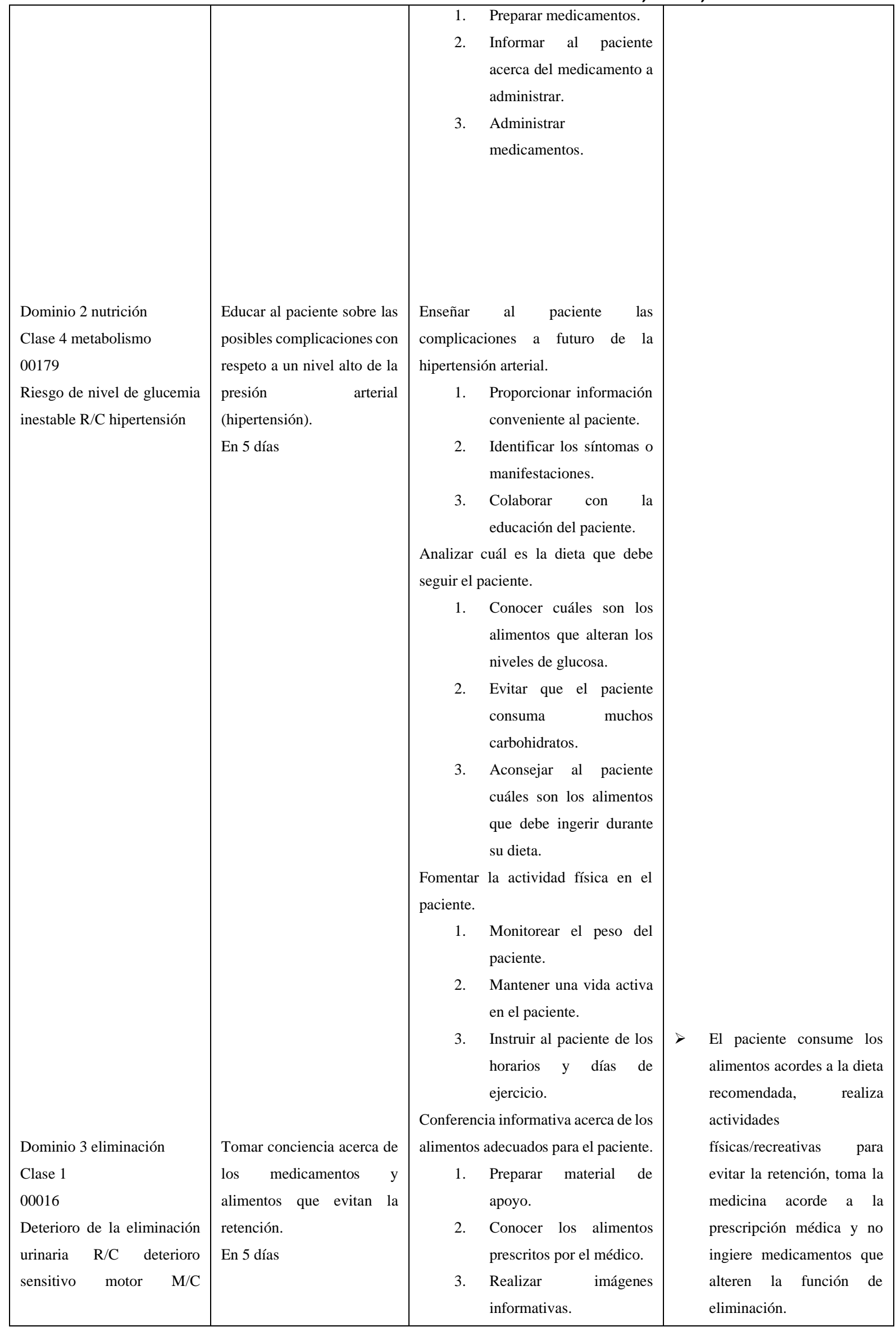




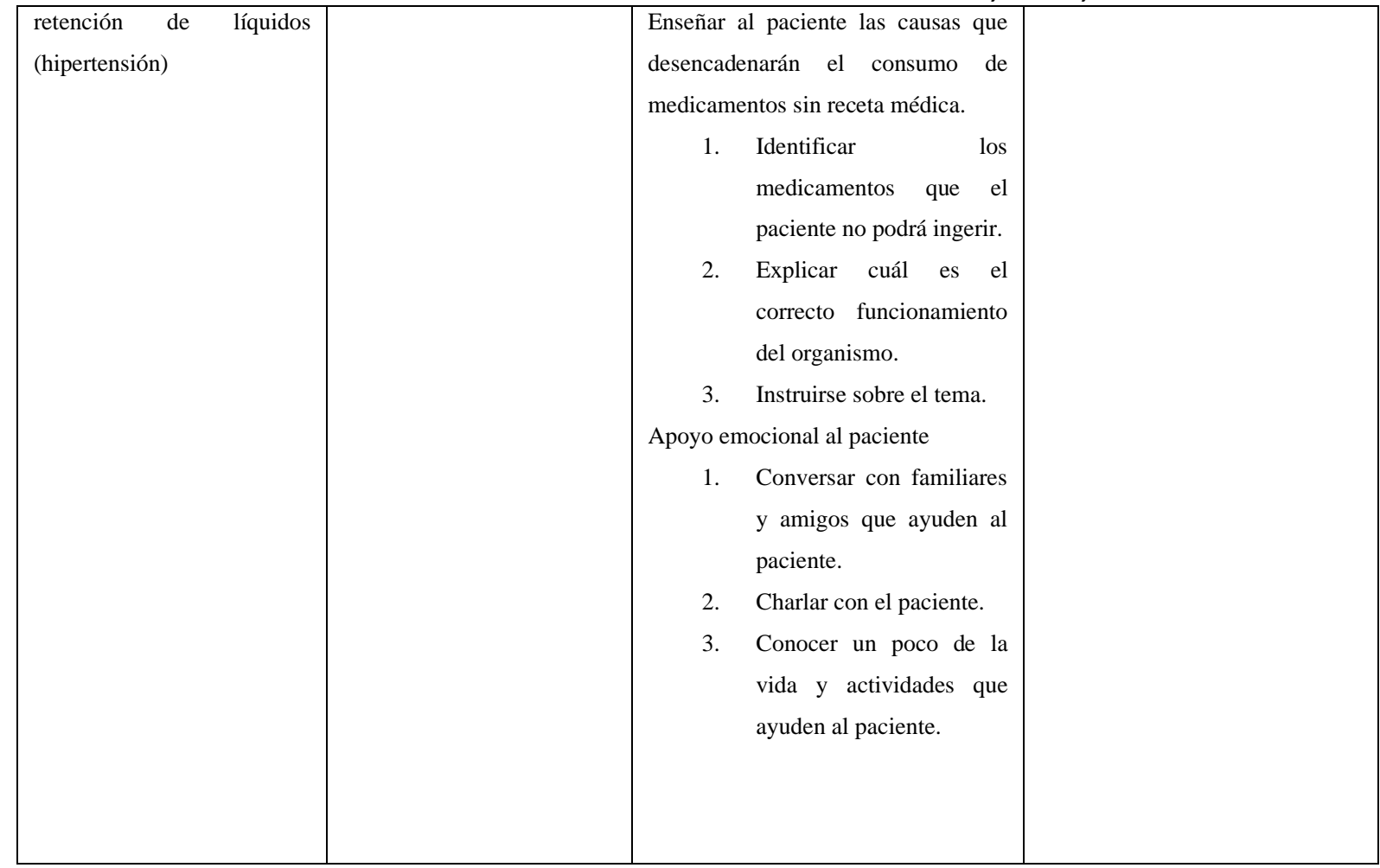

Elaborado por autores del artículo

\section{Conclusiones}

$>$ Mediante los aprendizajes adquiridos como resultado de la investigación fue posible conocer científicamente lo que engloba la hipertensión arterial y los principales factores de riesgo que causan esta enfermedad en la población adulta y cuáles son los efectos causados.

$>$ Durante la investigación realizada se identificaron las evidencias de la intervención de enfermería en el cuidado de adultos con hipertensión arterial basado en aspectos fundamentales para favorecer el bienestar del paciente y de tal manera dar a conocer los riesgos a los que se encuentran expuestos.

$>$ Con el proyecto integrador de saberes se puede destacar la importancia del presente trabajo, porque contribuye a enriquecer el conocimiento y aprendizaje en base a las asignaturas impartidas por los docentes en el presente semestre, las que han sido el principal apoyo para el desarrollo de este proyecto.

\section{Colaboradores de la Investigación:}

Álvarez Intriago Milton Asdrúbal; Álvarez Macías Josselyn Lisbeth; Bravo Valencia Dayse Jamileth; Cedeño Párraga Steban Xavier; Muñoz Cedeño Elvia Gissela; Muñoz Pacheco Blanca Elizabeth; Guaranda Pibaque Stephanny Jubileny; Pinargote Zamora Deliany Alejandra; Rundo Pichogagón Geovanna Elizabeth.

\section{Referencias Bibliográficas}

(2017). Recuperado el 14 de Agosto de 2020, de Ministerio de Salud Publica : https://www.salud.gob.ec/ministerio-de-salud-continuamente-emprende-acciones-para-prevenir-lahipertension-arterial/

(2019). Recuperado el 07 de Agosto de 2020, de Organizacion Mundial de la Salud: https://www.who.int/es/news-room/fact-sheets/detail/hypertension

Abad, T. (2016). Recuperado el 1 de Agosto de 2020, de Hospital general Universitario de Ciudad Real: http://www.hgucr.es/areas/area-de-enfermeria/planes-de- 
cuidados/\#: :text=Un\%20plan\%20de\%20cuidados\%20estandarizado,de\%20mejora\%20de\%20las\%20i ntervenciones.

Aguilar, J. L., \& Vega, S. M. (2020). Recuperado el 1 de Agosto de 2020, de Universidad tecnica de Babahoyo: http://dspace.utb.edu.ec/handle/49000/7965

Cabrera Perez, R. A. (2017). Recuperado el 07 de Agosto de 2020, de Reositorio Digital: http://repositorio.utmachala.edu.ec/handle/48000/9893

Cisne, G. J. (2020). Recuperado el 20 de Agosto de 2020, de Revista medica : https://revistamedica.com/atencion-de-enfermeria-modelo-marjory-gordon-esclerosis-lateralamiotrofica/

CONSEJO NACIONAL DE CIENCIA Y TECNOLOGÍA (CONACYT). (2019). Recuperado el 13 de Agosto de 2020, de CONSEJO NACIONAL DE CIENCIA Y TECNOLOGÍA (CONACYT): https://www.conacyt.gov.py/estudioindica-3-10-estudiantes-salud-hipertensos-culminar-carrera

Deborah, S., Pereyra, I. C., Cófreses, P., \& Dorina, S. (Junio de 2015). Estudio de las representaciones sociales de la hipertensión arterial según género. Scielo, 21(1).

https://secardiologia.es/comunicacion/notas-de-prensa/notas-de-prensa-sec/4293-raza-condiciona-riesgo-. (2014). Recuperado el 1 de Agosto de 2020, de Sociedad Española de Cradiología : https://secardiologia.es/comunicacion/notas-de-prensa/notas-de-prensa-sec/4293-raza-condicionariesgo-

línea, A. e. (2016). Recuperado el 20 de Agostp de 2020, de Aprende en línea: http://aprendeenlinea.udea.edu.co/lms/moodle/mod/page/view.php?id=61971

Montes de Oca, L. S., Guerra, N., Castañeda, M., \& Coma, N. (Marzo de 2019). Asociacion entre hipertensión y enfermedad peritonela. medigrapic.com, 11(S1).

Pérez, R. A. (2017). Proceso de atención de enfermería en los pacientes con hipertensión arterial para la prevención de complicaciones. Recuperado el 13 de Agosto de 2020, de Machala : Universidad Técnica de Machala: http://repositorio.utmachala.edu.ec/handle/48000/9893

Perez, S. C. (2017). Recuperado el 23 de Agosto de 2020, de Salud cardio: https://www.fbbva.es/microsites/salud_cardio/mult/fbbva_libroCorazon_cap12.pdf

Rybertt, T., Azua, E., \& Rybertt, F. (Julio de 2016). RETARDO DE CRECIMIENTO INTRAUTERINO: CONSECUENCIAS A LARGO PLAZO. SciencieDierct, 27(4).

Saniabra, J., \& Pérez, L. (Diciembre de 2013). Aplicación del proceso de atención de enfermería por estudiantes, un estudio desde la experiencia vivida. Scielo, 13(4).

Vignolo, J., Vacarezza, M., Álvarez , C., \& Sosa, A. (Abril de 2015). Niveles de atención, de prevención y atención primaria de la salud. Scielo, 33(1).

Wagner-Grau, P. (Diciembre de 2016). Fisiopatología de la hipertensión arterial. Scielo, 71(4).

yo, P. c. (2017). Recuperado el 1 de Agosto de 2020, de Pacientes como yo : http://pacientescomoyo.com.mx/hipertension-arterial/

Young, P., Hortis de Smith, V., Chambi , M., \& Finn, B. (Junio de 2011). Florence Nightingale (1820-1910), a 101 años de su fallecimiento. Scielo, 139(6). 